\title{
MICROSTRUCTURE AND OPTO-ELECTRONIC EFFECTS IN MXENES SPINCOATED FROM POLAR APROTIC SOLVENTS ON ITO
}

\author{
${ }^{1}$ Kezia SASITHARAN, ${ }^{1}$ Jaroslav KULICEK, ${ }^{2}$ Yaryna SOYKA, ${ }^{2}$ Michal PROCHAZKA, \\ ${ }^{2}$ Maria OMASTOVA, ${ }^{1}$ Bohuslav REZEK \\ ${ }^{1}$ Centre for Advanced Photovoltaics, Faculty of Electrical Engineering, Czech Technical University \\ in Prague, Prague, Czech Republic, EU \\ 2 Polymer Institute SAS, Bratislava, Slovakia, EU
}

https://doi.org/10.37904/nanocon.2021.4307

\begin{abstract}
MXenes have drawn considerable attention in the past decade, thanks to their attractive properties such as metallic conductivity and surface hydrophilicity. While MXenes form highly stable dispersions in water, it can act as a limitation for certain applications such as photoactive layer in photovoltaic devices. In this work, delaminated MXenes of aqueous solution type $\mathrm{Ti}_{3} \mathrm{C}_{2}$ were prepared first, and then we have used a solventexchange technique to prepare suspensions of MXenes in three polar aprotic solvents namely, DMSO, DMF and NMP. Upon spin-coating under the same conditions, each solvent variation yields a different thin film morphology - in terms of particle size and surface coverage, as evidenced from AFM investigations. While the MXenes in DMSO yielded large aggregated particles with $\mu \mathrm{m}$-sized islands in the film, MXenes in DMF and NMP were found to form films with well-dispersed MXene sheets in the size range $250 \mathrm{~nm}-50 \mathrm{~nm}$ and $80 \mathrm{~nm}$ $10 \mathrm{~nm}$, respectively. This study also provides additional insights into the microstructure and opto-electronic properties of the MXene thin films using correlative Raman microscopy and photoluminescence spectroscopy. The information provided by this study on the variation in the properties depending on the solvent used to process and spin-cast the films are important for evaluating MXenes in thin film device applications.
\end{abstract}

Keywords: MXenes, spin-coating, thin-film microstructure, atomic force microscopy, photoluminescence, polar aprotic solvents

\section{INTRODUCTION}

Ever since the successful exfoliation of a single layer graphene from graphite, there has been an increased interest in two-dimensional materials.[1] In recent years, two dimensional materials such as boron nitride, transition metal dichalcogenides, phosphorenes, co-ordination polymers such as metal-organic framework nanosheets (MONs) and coordination organic frameworks (COFs), have been explored and were found to possess exceptional opto-electronic and mechanical properties.[2] MXenes are a novel class of twodimensional materials that can be referred to with the general formula $M_{n+1} X_{n} T_{x}$ where $M$ is a transition metal, $X$ is carbon or nitrogen, and $T$ represents the surface functional groups $(-O H,-O$ and/or $-F)$.[3] MXenes are derived from inorganic ternary carbides or nitrides (referred to as the MAX phase, where A represents an Agroup element). The M-A bonds are more chemically reactive, and therefore the A-layer can be selectively etched upon reaction with hydrofluoric acid (HF).[3] This is followed by a de-lamination step in which molecules such as water, dimethyl sulfoxide (DMSO), tetrabutylammonium hydroxide (TBAOH), etc. are intercalated into the interlayer spacing thereby forcing the layers apart and generating single-flake MXene dispersions.[4] The quality of the produced sheets, their surface chemistry and structural defects depend on the etching conditions.[5] 
MXenes are highly conductive and therefore exhibit superior electrical and mechanical properties as compared to other low dimensional materials.[6] The functional groups on the surface make the MXenes hydrophilic and stable when stored as highly concentrated suspensions in water.[7] This enables the creation of MXene inks which can be conveniently stored, transported, and coated onto substrates. These properties have enabled the exploration of MXenes as conductive supports for photocatalysis, as photocatalysts for $\mathrm{H}_{2}$ production, as electrodes in Li-ion batteries and as micro-supercapacitors. [8] The numerous data available in the literature on the colloidal properties of MXenes in water are useful for these applications. While a lot of new research is focused on using antioxidants as additives to enhance the shelf-life and stability of MXenes in water, very limited studies exist about the evaluation of MXene thin-films cast from different solvents.[9] The lack of these studies limit the possibility of exploring MXenes in applications where water exposure is detrimental, such as in organic or perovskite solar cells.

In this work, we report a solvent-exchange technique replacing water with polar aprotic solvents - dimethyl sulfoxide (DMSO), dimethyl formamide (DMF), and N-methyl pyrrolidone (NMP) - and we explore the microstructure and optoelectronic properties of the thin films spin-coated from each of these solvents. We study the thin film morphology using atomic-force microscopy (AFM) and evaluate the topography and phase images to understand the distribution of the material on the substrate after spin-coating. We have used Raman spectroscopy as a tool for evaluating the structural composition of the films in combination with microstructure mapping which gives additional information about the film morphology. We also report photoluminescence spectra as an important tool in determining the opto-electronics of the films. We anticipate that this preliminary investigation into the thin-film morphology of MXene films when spin-coated from polar aprotic solvents can act as a guideline for applications where MXenes being stored in water can be a limiting factor.

\section{EXPERIMENTAL METHODS}

\subsection{Delaminated MXenes}

The starting material for the preparation of MXenes was a MXene paste obtained from Drexel University (internal batch labelling MX_201123_Drexel_paste). This paste was prepared from $\mathrm{Ti}_{3} \mathrm{AlC}_{2} \mathrm{MAX}$ phase using MILD etching method ( $\mathrm{HCl} / \mathrm{LiF}$ ) previously reported in literature.[3] Upon etching, an expanded MXene form is obtained, from which the single layers have to be separated. For delamination, the expanded MXenes were mixed with LiF followed by centrifugation to remove and collect single layered nanoparticles. For getting the required concentration, a vacuum concentrator (SpeedVac) was used to remove water and increase the concentration. This resulted in the $0.9 \mathrm{mg} / \mathrm{mL}$ dispersion of MXene in water used in this study.

\subsection{Solvent -exchange with polar aprotic solvents}

For solvent exchange, a known volume of the MXene-water sample was taken and centrifuged at $13000 \mathrm{rpm}$ for 30 minutes and the supernatant above the sediment was pipetted out. The sedimented MXenes were redispersed in the polar aprotic solvents - DMSO, DMF and NMP respectively and centrifuged at 13000 rpm for 5 minutes and the supernatant above the sediment was pipetted out. This process of addition of fresh solvent followed by centrifugation and pipetting out the supernatant liquid was performed for 3 cycles, to ensure the complete removal of any residual water. This repeated washing cycle can result in aggregation of the particles; therefore, the final sediment was re-dispersed in fresh solvent and sonicated at $37 \mathrm{kHz}$ for 30 minutes and stored for further analysis.

\subsection{Spin-coated films}

ITO-coated glass substrates obtained from Ossila Ltd were used for the film preparation. The films were prepared using a dynamic spin-coating technique where $50 \mu \mathrm{l}$ of the MXene dispersion was dropped onto the substrate, followed by a spin-coating at $1000 \mathrm{rpm}$ for 60 seconds, with acceleration of $200 \mathrm{rpm} / \mathrm{s}$. The 
substrates were then placed on a pre-heated hotplate at $100^{\circ} \mathrm{C}$ for 30 seconds for quick drying of any residual solvent. The substrates were then used for further characterization.

Delaminated MXenes dispersed in water

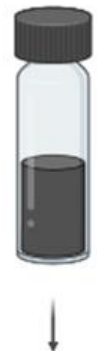

(2) Centrifuged off and re-dispersed in polar aprotic solvents DMSO, DMF, NMP

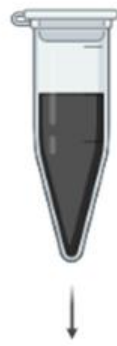

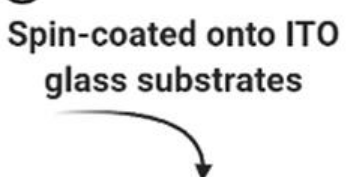

a. MXene in water

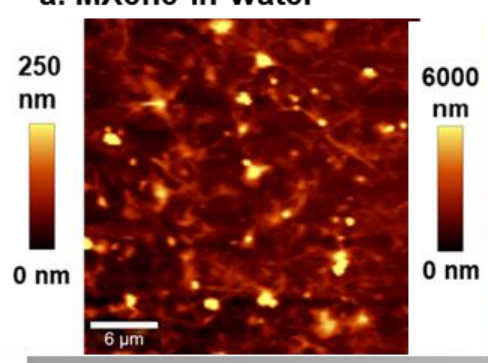

b. MXene in DMSO

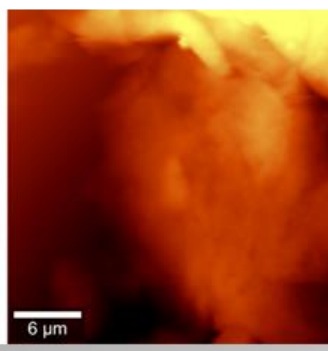

c. MXene in DMF

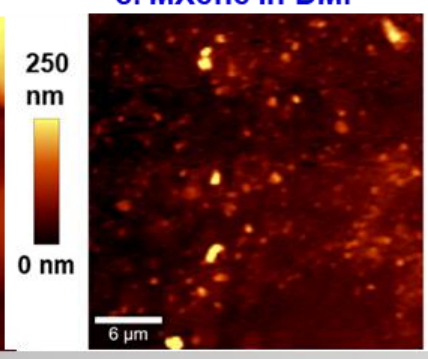

d. MXene in NMP

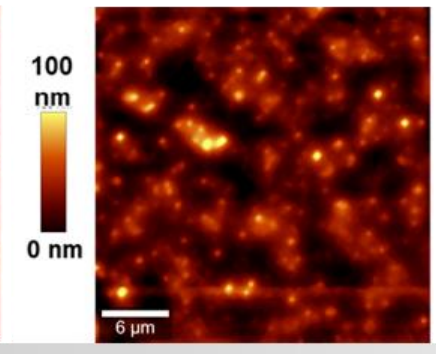

AFM Topography
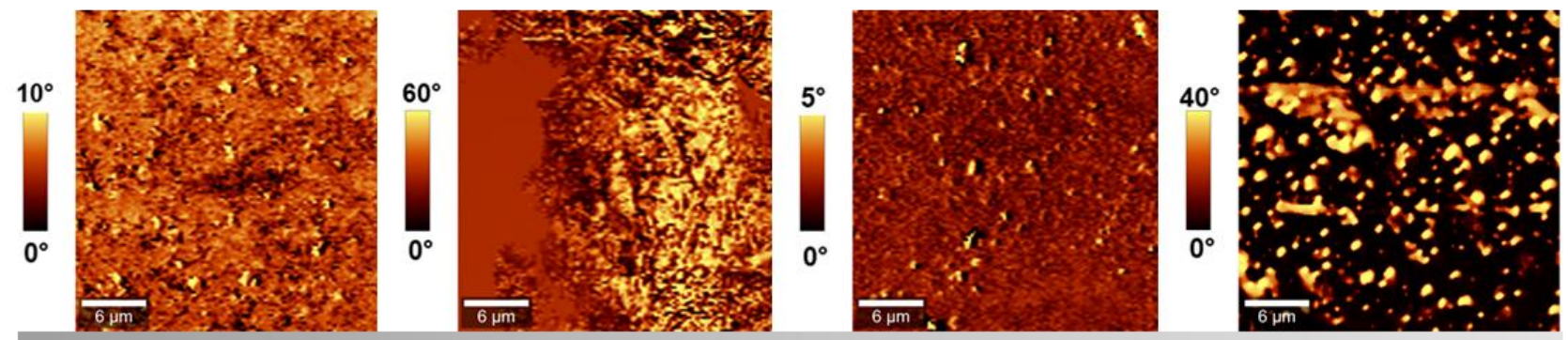

AFM Phase

Figure 1 Schematic representation of the processes used for sample preparation in this work and AFM topography and phase images of a representative $30 \times 30 \mu \mathrm{m}^{2}$ area of the samples: (a) MXene-Water, (b) MXene-DMSO, (c) MXene-DMF, and (d) MXene-NMP

\section{RESULTS AND DISCUSSION}

\subsection{Atomic Force Microscopy}

Atomic Force Microscopy (AFM) was used as an easy technique to analyze the MXene morphology in the films spin-coated from different solvents. The MXene-water film (Figure 1a) was found to be uniform with the sheets appearing in rolled or wrinkled morphology, interspersed with some larger particles of $\sim 200 \mathrm{~nm}$ height. In the film cast from MXene-DMSO (Figure 1b), there is a stark difference in increased aggregation of MXene particles ending up in $\mu \mathrm{m}$-sized islands on the surface. In the film cast from MXene-DMF and MXene-NMP (Figure $1 \mathbf{c , d}$ ) we did not observe such aggregation effects as in DMSO. The particles appear more uniformly distributed. The parameters evaluated from the AFM topography and phase data are summarized in Table 1. The average size of the particles with standard deviation in $\mathrm{nm}$ calculated from the $30 \times 30 \mu \mathrm{m}^{2}$ scan area shows that the size of particles decreases in the order MXene-DMSO > MXene-Water > MXene-DMF > 
MXene-NMP. When we compare the peak to peak roughness, which is the vertical difference between the highest and lowest points on the sample surface, we find that MXene-DMSO gives the highest value at $~ 3.7$ $\mu \mathrm{m}$, followed by MXene-water at $\sim 603 \mathrm{~nm}$, MXene-NMP at $\sim 133 \mathrm{~nm}$ and MXene-DMF at $\sim 72 \mathrm{~nm}$. These results show that MXene in DMF is an ideal solvent medium, resulting in more uniformly sized particle distribution when spin-coated, even better than in commonly used water. On the other hand, MXene in NMP results in a relatively higher number of smaller particles and high AFM phase contrast. Each of these samples have a different phase value than the MXene-water sample, which indicates that there is a variation in the surface properties (including chemistry) depending on the solvent from which the material was spin-coated.

Table 1 Comparison of the particle size, peak to peak roughness and RMS phase values between MXenewater, MXene-DMSO, MXene-DMF and MXene-NMP calculated from Figure 1

\begin{tabular}{|l|c|c|c|c|}
\hline Values from AFM & MXene-water & MXene-DMSO & MXene-DMF & MXene-NMP \\
\hline Average size of particles $(\mathbf{n m})$ & $75 \pm 49$ & $1153 \pm 934$ & $42 \pm 14$ & $36 \pm 15$ \\
\hline Roughness peak to peak (nm) & 603 & 3771 & 72 & 133 \\
\hline Phase peak to peak ( $\left.{ }^{\circ}\right)$ & 33 & 76 & 24 & 51 \\
\hline
\end{tabular}

\subsection{RAMAN MICROSTRUCTURE AND SPECTRUM}

a. MXene in water

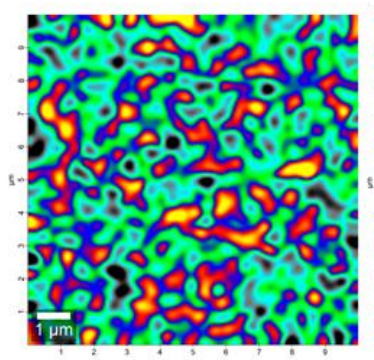

e.

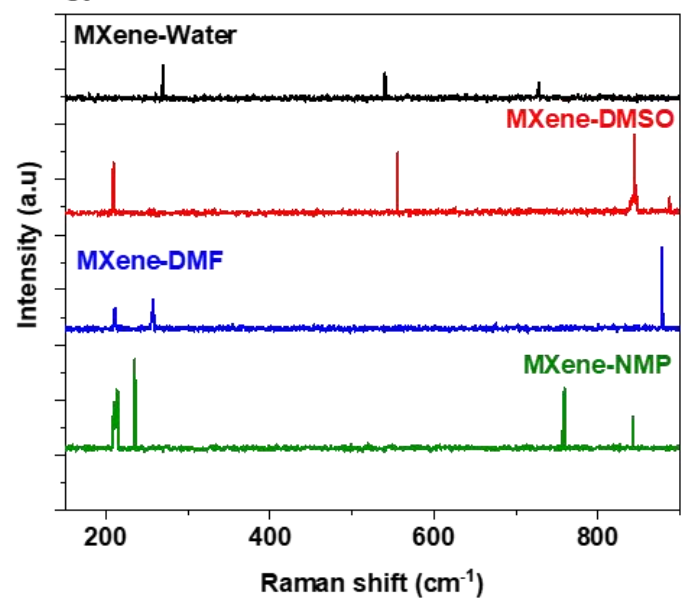

b. MXene in DMSO

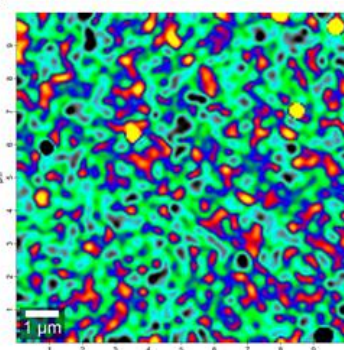

c. MXene in DMF

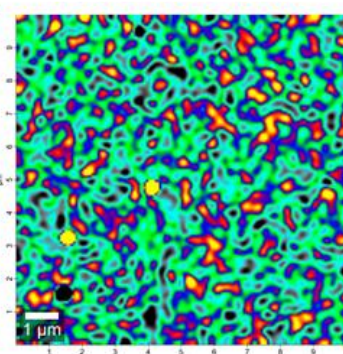

d. MXene in NMP
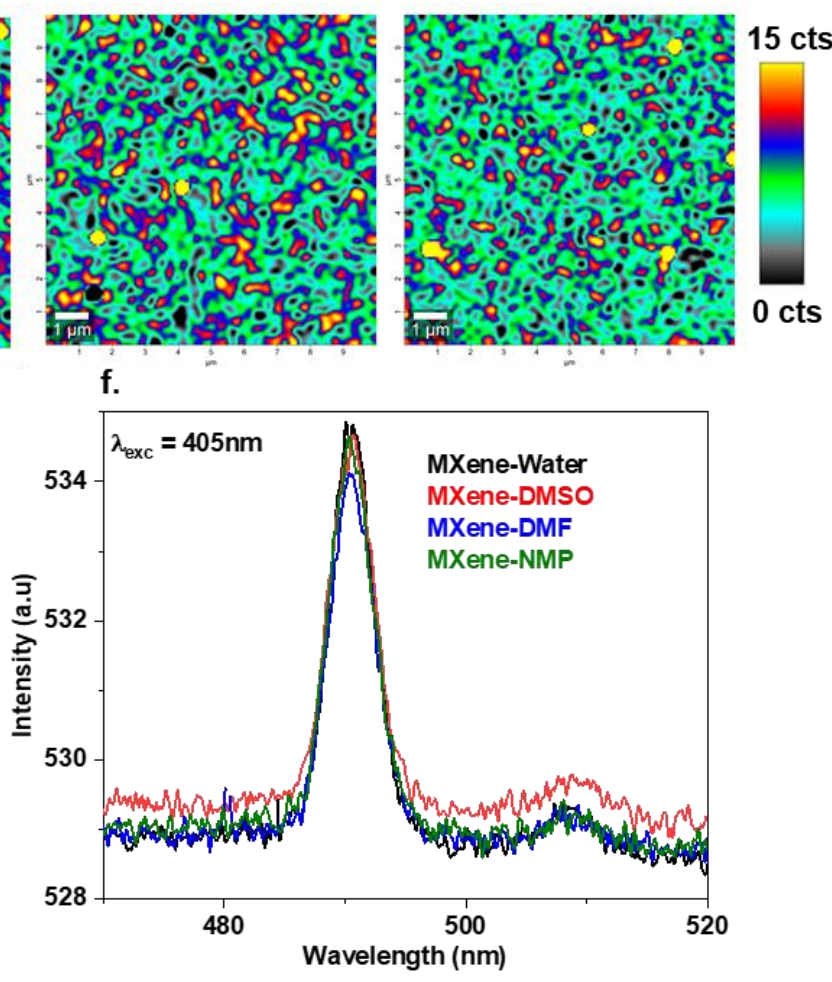

Figure 2 Raman microstructure of the -(a) MXene- Water; (b) MXene-DMSO; (c) MXene-DMF and (d) MXene-NMP films; (e) Acquired Raman single spectrum of the films (f) Photoluminescence spectrum of the samples

Raman spectroscopy has been used as a technique for MXene films to measure the lattice vibrations and provide information about bonding in the structure. When surface groups are added, it is expected that the number of atoms in the unit cell increases and therefore the number of vibrations increases accordingly resulting in an increased number of bands in the Raman spectra. Unit cell distortion and distribution of surface 
groups result in peak shifting and peak broadening as well. The peaks have been assigned based on the date from previously reported literature.[10] In the spectra reported below in Figure 2, the peak at $200 \mathrm{~cm}^{-1}$ corresponds to the $\mathrm{A} 1 \mathrm{~g}$ vibrations of the MXene flake region. The region between 230 and $470 \mathrm{~cm}^{-1}$ correspond to the in-plane E1g vibrations of the surface groups attached to the Ti atoms. We observe the appearance of new peaks in this region in the MXene-DMF and MXene-NMP. The region between 580 and $730 \mathrm{~cm}^{-1}$ corresponds to carbon vibrations. One of the issues with MXenes is that they degrade over time upon exposure to oxygen and moisture, resulting into degradation and formation of $\mathrm{TiO}_{2}$. It is worth noting that the typical Raman peaks of $\mathrm{TiO}_{2}\left(\mathrm{Eg}\right.$ at $150 \mathrm{~cm}^{-1} ; \mathrm{B} 1 \mathrm{~g}$ at $400 \mathrm{~cm}^{-1}, \mathrm{~A} 1 \mathrm{~g}$ at $600 \mathrm{~cm}^{-1}$ ) are missing from these films, probably due to its relatively low content in the films.[11] Figure 2 shows the Raman mapping of the film microstructure corresponding to the A1g vibrations in the MXene flake region. The size of the flakes or particles corresponding to the Raman vibrations are well correlated with the lateral sizes of the protrusions observed in the AFM topography of the MXene films. This confirms that the protrusions or dot-like structures in the AFM images are indeed small-sized MXenes and not $\mathrm{TiO}_{2}$ dots, and the varying phase contrast is due to the different surface chemistry introduced by the different solvents. The Raman analysis therefore shows the distinction in the molecular fingerprints of the differently processed films, confirms the absence of degradation products and gives a more detailed information about the MXene film microstructure.

\subsection{PHOTOLUMINESCENCE}

Figure $2 \mathrm{f}$ shows that our MXene films exhibit photoluminescence. While MXenes photoluminescence is in contradiction to their metallic band structure, such light emission has previously been attributed to a band gap enlargement effect induced by quantum confinement, applicable to small scale MXene quantum dots.[12] Larger flakes have also previously been reported to show photoluminescence.[13] Another possible mechanism for photoluminescence in MXenes is based on the theory that TiOx can form around Ti vacancies on the surface of $\mathrm{Ti}_{3} \mathrm{C}_{2} \mathrm{~T}_{x}$ MXene.[3],[4] While the Raman spectra do not show the typical peaks of $\mathrm{TiO}_{2}$, it is only an indication of the absence of $\mathrm{TiO}_{2}$ in large quantities, and is not enough evidence to rule out the possibility of $\mathrm{TiO}_{2}$ interspersed within the $\mathrm{Ti}_{3} \mathrm{C}_{2} \mathrm{~T}_{x}$ films. Investigating these films using $\mathrm{X}$-ray diffraction combined with photoluminescence mapping of the films to gather more insights into the photoluminescence mechanism will be part of our future studies.

\section{CONCLUSIONS}

Delaminated MXene sheets in aqueous media and MXenes processed in polar aprotic solvents (DMSO, DMF, NMP) were spin-coated onto ITO coated glass substrates and comparatively evaluated using AFM, Raman mapping and photoluminescence. MXenes spin-coated from water, DMF, and NMP formed uniform films, with particle sizes being even smaller and more uniform in the case of DMF and NMP as compared to water. On the other hand, MXenes spin-coated from DMSO gave rise to agglomerated particles with $\mu \mathrm{m}$-sized features. The Raman single spectra show the presence of all the characteristic Raman peaks of MXenes with distinctive differences in the number of bands and peak shifts. These differences may be attributed to the surface modifications induced by the different solvent environments. Raman mapping showed that the microstructure correlates with the topography observed in AFM. The films exhibit strong photoluminescence upon excitation under UV/Visible light, which can be attributed to either strong confinement effects induced by the small sized particles, or the presence of $\mathrm{TiO}_{2}$ defects around Ti vacancies in the MXene structure. Further characterization using XPS, FT-IR, XRD, PL, photo-Kelvin probe and APS will be our future work to determine the type of surface modification induced by the different solvents, its correlation with the film microstructure and the resulting effects on the photovoltage. We anticipate that these results will be useful for evaluating MXenes as electrodes, buffer layers or surface passivation layers in devices where use of water-based solvents is detrimental and better control of MXenes dispersions and chemistry may be beneficial. 


\section{ACKNOWLEDGEMENTS}

\section{The work has been supported by the Centre for Advanced Photovoltaics \\ (CZ.02.1.01/0.0/0.0/15_003/0000464) and from the European Union's Horizon 2020 project Nano2Day, grant agreement No. 777810.}

\section{REFERENCES}

[1] J. C. MEYER, A. K. GEIM, M. I. KATSNELSON, K. S. NOVOSELOV, T. J. BOOTH and S. ROTH. The structure of suspended graphene sheets. Nat. [online]. 2006, vol. 446, no. 7131, pp. 60-63. Available from: https://doi.org/10.1038/nature05545.

[2] K. SASITHARAN et al. Metal-organic framework nanosheets for enhanced performance of organic photovoltaic cells. J. Mater. Chem. A. [online]. Mar. 2020, vol. 8, no. 12, pp. 6067-6075. Available from: https://doi.org/10.1039/c9ta12313j.

[3] M. ALHABEB et al. Guidelines for Synthesis and Processing of Two-Dimensional Titanium Carbide (Ti3C2Tx MXene). Chem. Mater. [online]. Sep. 2017, vol. 29, no. 18, pp. 7633-7644. Available from: https://doi.org/10.1021/ACS.CHEMMATER.7B02847.

[4] L. H. KARLSSON, J. BIRCH, J. HALIM, M. W. BARSOUM, and P. O. Å. PERSSON. Atomically Resolved Structural and Chemical Investigation of Single MXene Sheets. Nano Lett. [online]. Aug. 2015, vol. 15, no. 8, pp. 4955-4960. Available from: https://doi.org/10.1021/ACS.NANOLETT.5B00737.

[5] X. SANG et al. Atomic Defects in Monolayer Titanium Carbide (Ti3C2Tx) Mxene. ACS Nano. [online]. Oct. 2016, vol. 10, no. 10, pp. 9193-9200. Available from: https://doi.org/10.1021/ACSNANO.6B05240.

[6] H. TANG, R. WANG, L. SHI, E. SHEREMET, R. D. RODRIGUEZ and J. SUN. Post-processing strategies for improving the electrical and mechanical properties of Mxenes. Chem. Eng. J. [online]. Dec. 2021, vol. 425, p. 131472. Available from: https://doi.org/10.1016/J.CEJ.2021.131472.

[7] B. AKUZUM et al. Rheological Characteristics of 2D Titanium Carbide (MXene) Dispersions: A Guide for Processing Mxenes. ACS Nano. [online]. Mar. 2018, vol. 12, no. 3, pp. 2685-2694. Available from: https://doi.org/10.1021/ACSNANO.7B08889.

[8] A. BHAT, S. ANWER, K. S. BHAT, M. I. H. MOHIDEEN, K. LIAO and A. QURASHI. Prospects challenges and stability of 2D MXenes for clean energy conversion and storage applications. npj 2D Mater. Appl. [online]. Jun. 2021, vol. 5, no. 1, pp. 1-21. Available from: https://doi.org/10.1038/s41699-021-00239-8.

[9] X. ZHAO et al. Antioxidants Unlock Shelf-Stable Ti3C2Tx (MXene) Nanosheet Dispersions. Matter. [online]. Aug. 2019, vol. 1, no. 2, pp. 513-526. Available from: https://doi.org/10.1016/J.MATT.2019.05.020.

[10] M. SHEKHIREV, C. E. SHUCK, A. SARYCHEVA, and Y. GOGOTSI. Characterization of MXenes at every step, from their precursors to single flakes and assembled films. Prog. Mater. Sci. [online]. Jul. 2021, vol. 120, p. 100757. Available from: https://doi.org/10.1016/J.PMATSCI.2020.100757.

[11] A. GRZEGÓRSKA et al. Enhanced photocatalytic activity of accordion-like layered Ti3C2 (MXene) coupled with Fe-modified decahedral anatase particles exposing $\left\{\begin{array}{lll}1 & 0 & 1\end{array}\right\}$ and $\left\{\begin{array}{lll}0 & 0 & 1\end{array}\right\}$ facets. Chem. Eng. J. [online]. Dec. 2021, vol. 426, p. 130801. Available from: https://doi.org/10.1016/J.CEJ.2021.130801.

[12] Q. XUE et al. Photoluminescent Ti3C2 MXene Quantum Dots for Multicolor Cellular Imaging. Adv. Mater. [online]. Apr. 2017, vol. 29, no. 15, p. 1604847. Available from: https://doi.org/10.1002/adma.201604847.

[13] L. ZHANG et al. Tuning the photoluminescence of large Ti3C2Tx MXene flakes. Ceram. Int. [online]. Jun. 2019, vol. 45, no. 9, pp. 11468-11474. Available from: https://doi.org/10.1016/J.CERAMINT.2019.03.014. 